\title{
Differential Expressions of Nerve Fibers During Distraction Osteogenesis - A Study in Rat Model
}

\author{
Jian Li"\#, Peter Zachrisson ${ }^{2 \#, ~ A n d r e ~ S t a r k ~}{ }^{3}$, Ulf Nannmark ${ }^{2}$, Hans Mark², Mahmood Ahmed ${ }^{4}$ and Aisha \\ S Ahmed ${ }^{1 *}$ \\ ${ }^{1}$ Department of Molecular Medicine and Surgery, Karolinska Institute, Sweden. \\ ${ }^{2}$ Department of Plastic Surgery, University of Gothenburg, Sweden. \\ ${ }^{3}$ Department of Clinical Sciences, Karolinska Institute, Sweden.
}

${ }^{4}$ Department of Neurobiology, Care Sciences and Society, Karolinska Institute, Sweden.

${ }^{\#}$ Authors Contributed Equally

*Corresponding author: Aisha Ahmed, Department of Molecular Medicine and Surgery, Karolinska Institutet, 17176, Stockholm, Sweden

\section{ARTICLE INFO}

Received: 幽 October 31, 2019

Published: 幽 November 12, 2019

Citation: Jian Li, Peter Zachrisson, Andre Stark, Ulf Nannmark, Hans Mark, Mahmood Ahmed, Aisha S Ahmed. Differential Expressions of Nerve Fibers During Distraction Osteogenesis - A Study in Rat Model. Biomed J Sci \& Tech Res 22(4)2019. BJSTR. MS.ID.003797.

Keywords: Nerve Regeneration; Substance P; Calcitonin Gene Related Peptide; Distraction Osteogenesis

\section{ABSTRACT}

Background: Nerve regeneration at fracture site is considered important for new bone formation. Present study was designed to investigate the innervation pattern during various stages of bone formation by using a rat model of distraction osteogenesis.

Methods: Total 63 male Sprague-Dawley rats were included in the study. A transverse osteotomy was performed to the right femur and an external fixator was applied. Femurs were distracted at the rate of $0.2 \mathrm{~mm} /$ day for 28 days for the total length of $8.4 \mathrm{~mm}$. Bone formation was monitored by radiographic analysis at the distracted area. Runx2, Taca and Cgrp gene expression was analyzed by the quantitative RT-PCR while GAP-43, PGP 9.5, SP, and CGRP expression was studied by immunohistochemistry (IHC) at day 7, 14, 21, 28, 42 and 56 postsurgery in the distraction portion of femur.

Results: Our radiographic analysis revealed new bone formation at day 7 and mature bone at day 56 in the distracted area of the fractured femur. An increased Runx2 mRNA levels between day 14 to 52, and up-regulated Sp and Cgrp gene expression was observed at all studied time points in the distracted compared to the intact control femur. GAP-43 immunoreactivity was increased at day 7, 14, 21 and 56, while PGP 9.5 at day 56 in the distracted femur. Increased SP immunoreactivity was observed at day 28 and 42. Up-regulated CGRP expression at day 14 while at day 21, CGRP immunoreactivity was down-regulated in the distracted compared to the intact control femur

Conclusion: Differential expressions of GAP-43, PGP 9.5, SP, and CGRP indicate pertinent role of nerve regeneration and sensory neuropeptides in bone formation during distraction osteogenesis.

\section{Introduction}

Distraction osteogenesis (DO) is a surgical technique used to treat musculoskeletal disorders from early childhood to middle age [1]. Osteogenesis is induced between bone ends under stable mechanical environment and when distraction is stopped, the newly formed bone in the gap slowly consolidates [2]. DO is a valuable tool to study biological processes of bone healing, from new bone formation to bone remodeling and maturation [3]. Mechanical forces applied in DO initiate a cascade of biologic processes of cellular differentiation, angiogenesis, mineralization of bone matrix and bone remodeling involving numerous growth factors $[4,5]$. 
However, mechanisms by which mechanical stimuli are registered and conveyed to bone formation are unknown. The findings of intense nerve regeneration at fracture site during fracture healing and the presence of neuropeptidergic receptors on osteoblasts suggest an active role of nervous system in bone formation in experimental studies [6,7]. In addition, skeletal sensory neurons are reported to produce various neurotransmitters important for bone metabolism [8].

Thus, sensory neuropeptides such as substance P (SP) and calcitonin gene related peptide (CGRP) identified in bone are reported to be involved in bone metabolism through a receptor mediated mechanism $[9,10]$. It has been shown that CGRP released by the nerve terminals innervating bone tissues binds to receptors expressed by osteoblasts and influence the synthesis of growth factors, cytokines and collagen synthesis facilitating bone formation [11-13]. Similarly, SP has been shown to stimulate various cellular processes important in bone formation such as osteogenic activity, osteoclast differentiation and resorption activity [14]. Osteoblasts are reported to be equipped with receptors to sensory neuropeptides SP and CGRP [15]. SP was shown to accelerate bone healing when injected into gap of rat mandible bone during mandibular bone distraction [16]. However, the knowledge behind the specific roles of SP and CGRP at different stages of bone formation during distraction osteogenesis is scarce. To our knowledge, limited numbers of studies have been designed to analyze the occurrence and distribution of nerve regeneration/ nerve maturation and sensory nerve fibers containing SP and CGRP in bone formation during bone distraction. The aim was to investigate nerve regeneration by studying the expression of gene associated protein-43 (GAP-43), mature nerves by protein gene product 9.5 (PGP 9.5) and sensory neuropeptides SP and CGRP, at different stages of bone formation in a rat model of distraction osteogenesis. As a marker of bone formation in distracted bone, we further studied the Runx2 gene expression in distracted bone.

\section{Material and Methods}

\section{Study Design}

The study included 63,10 weeks old male Sprague-Dawley rats with the body weight 350-400 grams. All animals were housed at $21^{\circ} \mathrm{C}$ in a 12-hour light/dark cycle with pellets and water ad lib according to the University of Gothenburg protocol. All experiments were approved by the local Committee for Animal Research and Ethics and conducted in accordance with the University's protocols. An osteotomy of the right femur was performed on 54 rats, while 9 rats served as intact controls. For DO, an external fixator was applied to right femur according to procedure described previously by us $[17,18]$. Based on previous findings, distraction was started at day 7 post-surgery at the rate of $0.2 \mathrm{~mm} / 12 \mathrm{~h}[19,20]$. Nine rats each were killed at day $7,14,21,28,42$ and 56 post-osteotomy with a distraction of $0,2.8,5.6,8.4$, and $8.4 \mathrm{~mm}$. The distraction at $8.4 \mathrm{~mm}$ was also studied at 14 and 24 days' consolidation period
(Table 1). Nine rats without fracture served as control and were euthanized at day 7 of the experiment.

Table 1: The experimental setup.

\begin{tabular}{|c|c|c|c|c|c|c|c|}
\hline Days & $\mathbf{0}$ & $\mathbf{7}$ & $\mathbf{1 4}$ & $\mathbf{2 1}$ & $\mathbf{2 8}$ & $\mathbf{4 2}$ & $\mathbf{5 6}$ \\
\hline & Surgery & & & & & & \\
\hline Radiography & & + & & + & + & & + \\
\hline Immunohistochemistry & & + & + & + & + & + & + \\
\hline Quantitative RT-PCR & & + & + & + & + & + & + \\
\hline
\end{tabular}

\section{Surgery}

Animals were anesthetized with 5\% isoflurane. Under sterilized conditions, a curved incision was made through the skin, running from the base of the tail to the knee. A skin flap was dissected from the underlying fascia. The femur was exposed by gentle dissection between the quadriceps and hamstring muscles, from the greater trochanter to the supracondylar region of the knee. Four holes $(0.7$ $\mathrm{mm}$ ) were drilled corresponding to pin diameters of $1.0 \mathrm{~mm}$ under a drill guide on the lateral aspect of the femur. Pins were inserted into the drill holes and screwed through the bone until their tips reached the outer surface of the far cortex. The four pins were canullated through the skin flap and the fixator was fastened to the pins at a preset distance from the bone surface. Between the two middle pins, an osteotomy was performed with a reciprocating saw under irrigation as reported previously $[17,18]$. The periosteum was re-approximated, and the wound closed. Unrestricted weight bearing and activity was allowed post-operatively.

\section{Radiographic Assessment}

Lateral views of distracted femur were taken at day 7, 21, 28 and 56 (Table 1) under inhalation anaesthesia (3-5\% isoflurane) by using dental X-ray machine (Siemens AG, Germany) with 1.25 second exposure time and $56 \times 76 \mathrm{~mm}$ X-ray films (Eastman Kodak Company, USA). Radiographic measurements were done manually on magnified printout of X-ray pictures. After modifying projection angle, bone healing was evaluated by calculating the callus index. The length of the widest part of the callus was determined in proportion to the diameter of the diaphysis measured from the intact site.

\section{Quantitative RT-PCR}

Rats (5 in each group) were anaesthetised with sodium pentobarbitone (60 mg/kg, intraperitoneal) and killed by decapitation. The distracted segments from femur were removed and immediately frozen in liquid nitrogen. Frozen tissues were homogenized by Mikro-dismembrator (B. Braun Biotech Int., Germany) and dissolved in 2-3 volumes of Trizol reagent (Invitrogen Life Technologies Inc., USA). RNA was then extracted and further purified using the RNeasy ${ }^{\circledR}$ MiniKit (Qiagen, USA) following the manufacturers protocol. Spectrophotometric analysis of the samples consistently showed absorption ratio at A260/280 $\mathrm{nm}=1.8-2.2$ indicating excellent purity of the RNA. First-strand 
cDNA were synthesized from $1 \mu \mathrm{g}$ of total RNA using the firststrand cDNA Synthesis Kit (Roche, Germany). Quantification assays were performed to detect the relative Sp, Cgrp and Runx2 mRNA expression. $3 \mu \mathrm{l}$ of each cDNA was used with TaqMan $1 \mathrm{X}$ Universal PCR Master Mix (Applied Biosystems, Roche) and run on ABI Prism 7300 Sequence Detection System. The hypoxanthine phosphorybosyl transferase (Hprt) was amplified as endogenous reference gene to normalize the quantification of target genes. The following Assay on Demand Kits (Applied Biosystems) was used Sp, Rn01500397_m1; Cgrp, Rn00569199_m1; Runx2, Rn01512296_ m1 and Hprt, Rn01527838_g1. Standard curve was established by plotting the Ct values against total RNA of internal control added to the reverse transcription reaction.

\section{Immunohistochemistry}

Rats (4 in each group) were anaesthetized with sodium pentobarbitone $(60 \mathrm{mg} / \mathrm{kg}$, inraperitonial) and perfused with 0.01 mol/l phosphate buffered saline pH (PBS) followed by Zamboni's fixative consisting of $4 \%$ paraformaldehyde in $0.2 \mathrm{~mol} / \mathrm{l}$ Sörensen phosphate buffer, $\mathrm{pH} 7.3$, containing $0.2 \%$ picric acid. Right femurs were removed and fixed in Zamboni fixative for two days at $4^{\circ} \mathrm{C}$ before subjecting to demineralization at room temperature in a solution containing cacodylate buffered 4\% ethylene diaminotetra acetic acid (EDTA) solution at $\mathrm{pH} 7.3$ for approximately 4 weeks, as described previously (33). Decalcified femur was soaked in $20 \%$ sucrose in $0.1 \mathrm{~mol} / \mathrm{l}$ Sörensen phosphate buffer, pH 7.2 , containing sodium azide and bacitracin (Sigma Chemicals, Sweden) for two days. Each demineralized femur portion was than divided sagittally in two halves and $1.5 \mathrm{~cm}$ long samples of the medial half of diaphysis, which included the proximal, middle and distal parts of the healing fracture. Thus, the regions undergoing endochondral and intramembranous ossification were all included in the same tissue section.

Subsequently, tissue sections were obtained at a thickness of $15 \mu \mathrm{m}$ using a Leitz ${ }^{\circledR} 1720$ cryostat (Ernst Leitz, Germany) and mounted on SuperFrost/Plus slides for immunohistochemistry analysis. Two sections, one close to the middle and other close to medial part of femur were chosen for immunostaining at each time point. Sections were hydrated in phosphate buffer for 5 minutes and with 5\% normal goat serum for 30 minutes at room temperature. Sections were later incubated overnight in a humid atmosphere with polyclonal antibody to GAP-43 (1:2000, catalog number: 1379011; Chemicon International, USA) and PGP 9.5 (1:10000, catalog number: MA1-20152 Chemicon International, USA), SP (1:10000, catalog number: T-4107; BACHEM Peninsula Laboratories, USA) and CGRP (1:10000, catalog number: IHC 6006; BACHEM Peninsula Laboratories, USA) at room temperature. After rinsing in phosphate buffer $(3 \times 5 \mathrm{~min})$ sections were incubated with biotinylated secondary goat anti-rabbit antibodies for 40 mins. Finally, the fluorochrome Cy2-conjugated avidin (1:1000, Amersham Life Science Inc., USA) was used for the visualization of the immunoreaction. An epifluoresence microscope (Eclipse E800,
Nikon, Japan) was used for fluorescence microscopic analysis. Images were captured by a video camera (DEI 750; Optronics Engineering, CA) attached to the microscope and stored in a computer. For semi-quantitative assessment, whole fracture area including the callus and the intact bone proximally and distally to the fracture was examined. From two consecutive tissue sections, 4 fields were selected from periosteum, bone marrow and connective tissue in and adjacent to distraction area, respectively, for quantification. A standard lower and upper threshold of fluorescence intensity was consistently applied for positively stained nerve fibers. The nerve fiber density was quantified by computerized image analysis program (Bergstrom Instruments, Sweden).

\section{Statistical Analysis}

The significance of the differences between experimental and control groups was analyzed by one-way analysis of variance (ANOVA), followed by Fisher's protected least significant difference test for quantitative variables. Significance was set at $\mathrm{P} \leq 0.05$. All data presented as mean \pm SEM.

\section{Results}

\section{Radiography}

Our radiographs revealed signs of new bone formation at day 7 and 21 in the distracted gap of fractured femur (Figure $1 \mathrm{a} \& 1 \mathrm{~b}$ ). After the desired length of distraction $(8.4 \mathrm{~mm})$ was achieved at day 28 the callus was filled with the radiolucent union (Figure 1c) and at day 56 was converted into mature bone bridging the gap in fractured femur (Figure 1d). Callus index indicated callus formation from day 7 to 56 indicating healing process (Figure 1e).

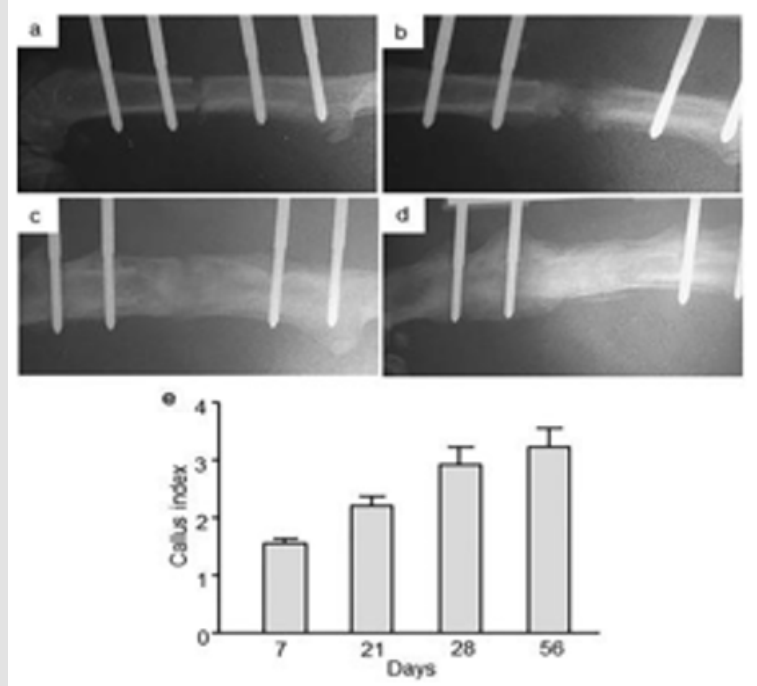

Figure 1: Radiographs representing lateral view of femur at a) day 7 with $0 \mathrm{~mm}$ distraction b) day 21 with $5.6 \mathrm{~mm}$ distraction c) day 28 with $8.2 \mathrm{~mm}$ distraction and d) day 56 with $8.4 \mathrm{~mm}$ distraction and 28 days of consolidation. Radiographs are representatives of 4 rats per group. e) Callus index of distracted portions of rat femur during distraction osteogenesis. Values are the mean \pm SEM of 4 rats per group. 


\section{Runx2 Gene Expression}

The RT-qPCR analysis revealed Runx2 gene expression in the intact control as well in the distracted area of femur at all studied time points (Figure 2). An up-regulated Runx2 mRNA levels were observed in the distracted portions of femur at day 14, 21, 28,
42 and 56 in comparison to intact control femur with 2 fold [p = $0.0001]$ increase at day $14,1.7$ fold increase $[p=0.0003]$ at day 21 , 1.9 fold increase $[p=0.0001]$ at day 28, 1.4 fold $[p=0.002]$ at day 42 and, 1.5 fold increase [ $p=0.04$ ] at day 56 . No statistically significant changes in Runx2 mRNA levels were obvious at day 7 in the distracted compared to intact control femur (Figure 2).

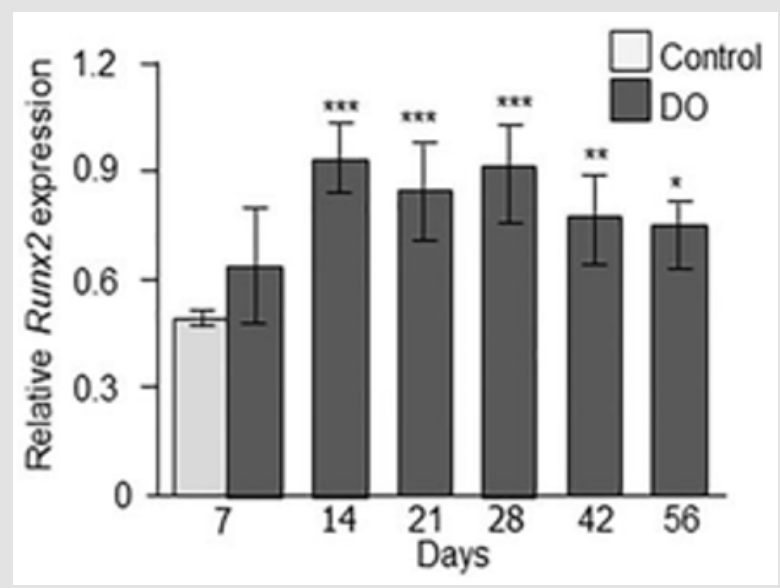

Figure 2: Relative Runx2 gene expression at day 7, 14, 21, 28, 42, and 56 from intact control (only day 7) and distraction osteogenic (DO) femur. Values are expressed as mean \pm SEM of 5 rats per group. ${ }^{*} \mathrm{P} \leq 0.05$, ${ }^{* * \mathrm{P}} \leq 0.005$ and ${ }^{* * *} \mathrm{P} \leq 0.0005$, compared to intact controls by ANOVA followed by Fisher's protected least significant difference test.

\section{Gene Associated Protein-43 (GAP-43) And Protein Gene Product 9.5 (PGP 9.5) Imunoreactivity}

Immunohistochemical analysis showed nerve fibers positive to GAP-43 and PGP 9.5 in the distracted portion of femur at all studied time points with differential expression.
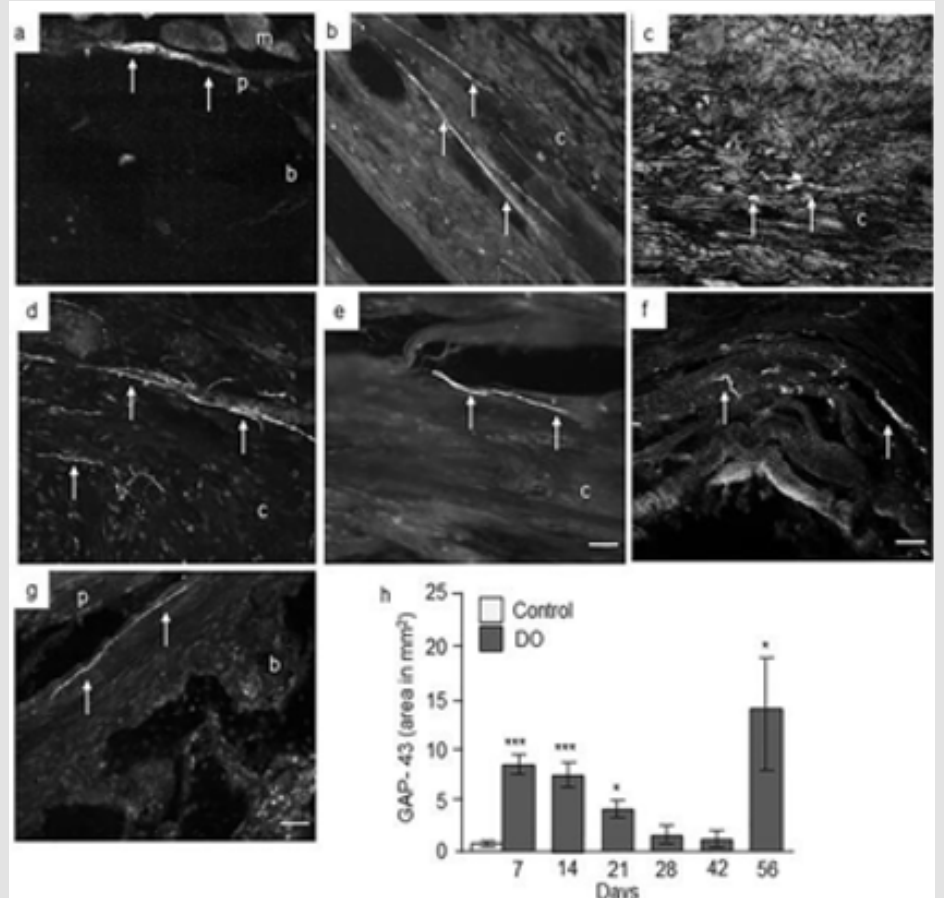

Figure 3: Immunofluorescence micrographs and semi-quantitative analysis of GAP-43 in intact and distracted portion of rat femur. Photomicrographs of a) intact control, and b) distracted femur at day 7; c) day 14; d) day 21; e) day 28; f) day 48, and $\mathrm{g}$ ) day 56. Original magnification is $20 \mathrm{x}$ and bars $=100 \mu \mathrm{m} .(\mathrm{m}=$ muscle, $\mathrm{p}=$ periosteum, $\mathrm{b}=\mathrm{bone}, \mathrm{c}=\mathrm{callus})$. h) Semiquantitative analysis of GAP-43 immunoreactivity (immunofluorescent area in $\mathrm{mm}^{2}$ ) at different time points from intact control and distraction osteogenic (DO) femur. Values are expressed as mean \pm SEM of 4 rats per group. ${ }^{*} \mathrm{P} \leq 0.05$ and ${ }^{* * *} \mathrm{P} \leq 0.0005$ compared to intact controls by ANOVA followed by Fisher's protected least significant difference test. 
GAP-43 Expression: Analysis of intact control femur demonstrated no immunoreactivity within the bone or in muscles. While, thin nerve fibers positive to GAP-43 were present at the periosteum at day 7 (Figure 3a). In the distracted femur, abundant GAP-43 positive nerve fibers were seen sprouting into the callus from hypertrophic periosteum close to osteotomy as well as in the periosteum and surrounding muscle fibers at day 7 and 14 postsurgery (Figure 3b \& 3c). GAP-43 positive staining was also seen in cells in the distracted area at day 14 (Figure 3c). At day 21, intense GAP43 immunoreactivity was observed in the periosteum, surrounding muscles, proximal intact bone as well as in the distraction area which apparently decreased at day 28 and 42 both for number of positive nerve fibers and intensity of the staining. A strong GAP-43 immunoreactivity was observed in and around the distracted gap at day 56 (Figure 3d-3g). Computerized image analysis demonstrated a 5 times increase in the GAP-43 immunoreactivity [p = 0.0005] at day 7, 4 times increase $[p=0.0005]$ at day 14 post-surgery and 74 $\%$ increase $[p=0.05]$ at day 21 compared to intact control rat femur. At days 28 and 42 no increase in GAP-43 immunoreactivity was observed. A significant 6 times increase [p $=0.05]$ in GAP-43 immunoreactivity was observed at day 56 in distracted compared to intact femur controls (Figure 3h).

PGP 9.5 Expression: Analysis of intact control femur demonstrated no immunoreactivity within the bone. However, very thin nerve fibers positive to PGP 9.5 were observed in the periosteum at day 7 (Figure 4a). At day 7 and 14 post-surgery, no nerve fibers were identified in the hematoma or surrounding muscle fibers while bundles of non-vascular nerve fibers positive to PGP 9.5 were seen in the hypertrophic periosteum and in connective tissues close to osteotomy (Figure $4 \mathrm{~b} \& 4 \mathrm{c}$ ). At day 21, very weak PGP 9.5 positive nerve fibers were identified (Figure $4 d$ ) while at day 28, thin PGP 9.5 positive nerve fibers were observed in the periosteum, in and around distracted gap and surrounding muscles fibers. At days 42 and 56 nerve fibers were seen close to the distraction area, in the bone marrow and surrounding muscles, and in periosteum some around the blood vessels (Figure $4 \mathrm{e}-4 \mathrm{~g}$ ). Computerized image analysis demonstrated no changes in PGP 9.5 immunoreactivity at days $7,14,28$ or 42 compared to intact rat femur. However, a significant increase [ 4 fold, $p=0.05$ ] was observed at day 56 compared to intact control rat femur (Figure $4 \mathrm{~h}$ ).
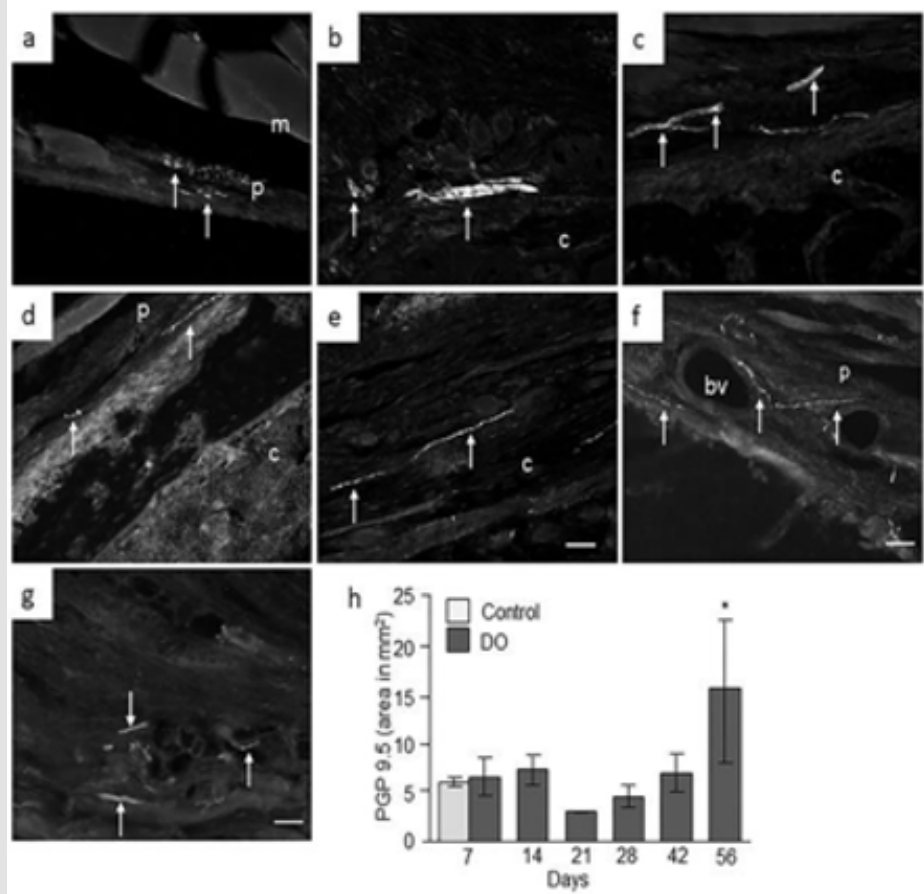

Figure 4: Immunofluorescence micrographs and semi-quantitative analysis of PGP 9.5 in intact and distracted portion of rat femur. Photomicrographs of a) intact control, and b) distracted femur at day 7; c) day 14; d) day 21; e) day 28; f) day 48, and g) day 56. Original magnification is $20 \mathrm{x}$ and bars $=100 \mu \mathrm{m} .(\mathrm{m}=$ muscle, $\mathrm{p}=$ periosteum, $\mathrm{c}=\mathrm{callus}, \mathrm{bv}=\mathrm{blood}$ vessel $) . \mathrm{h})$ Semi-quantitative analysis of PGP 9.5 immunoreactivity (immunofluorescent area in $\mathrm{mm}^{2}$ ) at different time points from intact control and distraction osteogenic (DO) femur. Values are expressed as mean \pm SEM of 4 rats per group. ${ }^{*} \mathrm{P} \leq 0.05$, compared to intact controls by ANOVA followed by Fisher's protected least significant difference test.

\section{Substance P (SP) and Calcitonin Gene Related Peptide} (CGRP) Expression

Our quantitative RT-PCR analyses showed that measurable levels of $S p$ and Cgrp mRNA were present in distracted and intact femur collected at all studied time points which were confirmed by IHC analysis.

SP Expression: Our RT-qPCR analyses of distracted portions of femur revealed a 6 fold $[p=0.0001]$ increase in Sp gene expression 
at day 7,5 fold $[p=0.0005]$ increase at day 14,6 fold $[p=0.0001]$ increase at day 21 , and 7 fold increase $[p=0.01]$ at day 28 compared to intact femur collected from control animals. Similarly, 2 fold increase [p $=0.007$ ] at day 42 and, 4 fold increase [ $p=0.03$ ] at day 56 in $S p$ mRNA levels were observed in distracted femur compared to intact control femur (Figure 5a). IHC analysis of intact control femur demonstrated no immunoreactivity within the bone. Thin, non-vascular nerve fibers positive to SP were observed at the periosteum at day 7 (Figure 5b). At days 7 and 14 abundant SP positive nerve fibers were present in the hypertrophic periosteum close to the osteotomy as well as in surrounding muscle fibers (Figure $5 c \& 5 d$ ). At day 21, a very weak SP immunoreactivity, with less number of SP-positive nerve fibers was observed in periosteum, surrounding muscles, proximal intact bone as well as at days 28 and 42 in these areas. Contrary to nerve fibers, we constantly observed SP- positive cell in and around the callus on these time points. A strong SP immunoreactivity was again observed in and around distracted gap at day 56 as well as cells positive to SP (Figure 5e-5h). Computerized image analysis demonstrated no significant change in the SP immunoreactivity in the distracted portion of femur at days 7, 14 or 21 compared to intact control rat femur. At days 28 and 42 there was $27 \%[p=0.05]$ and $39 \%$ [p = 0.005] increase in SP immunoreactivity respectively. At day 56 no difference in SP immunoreactivity was observed in distracted femur compared to intact femur controls (Figure 5i).

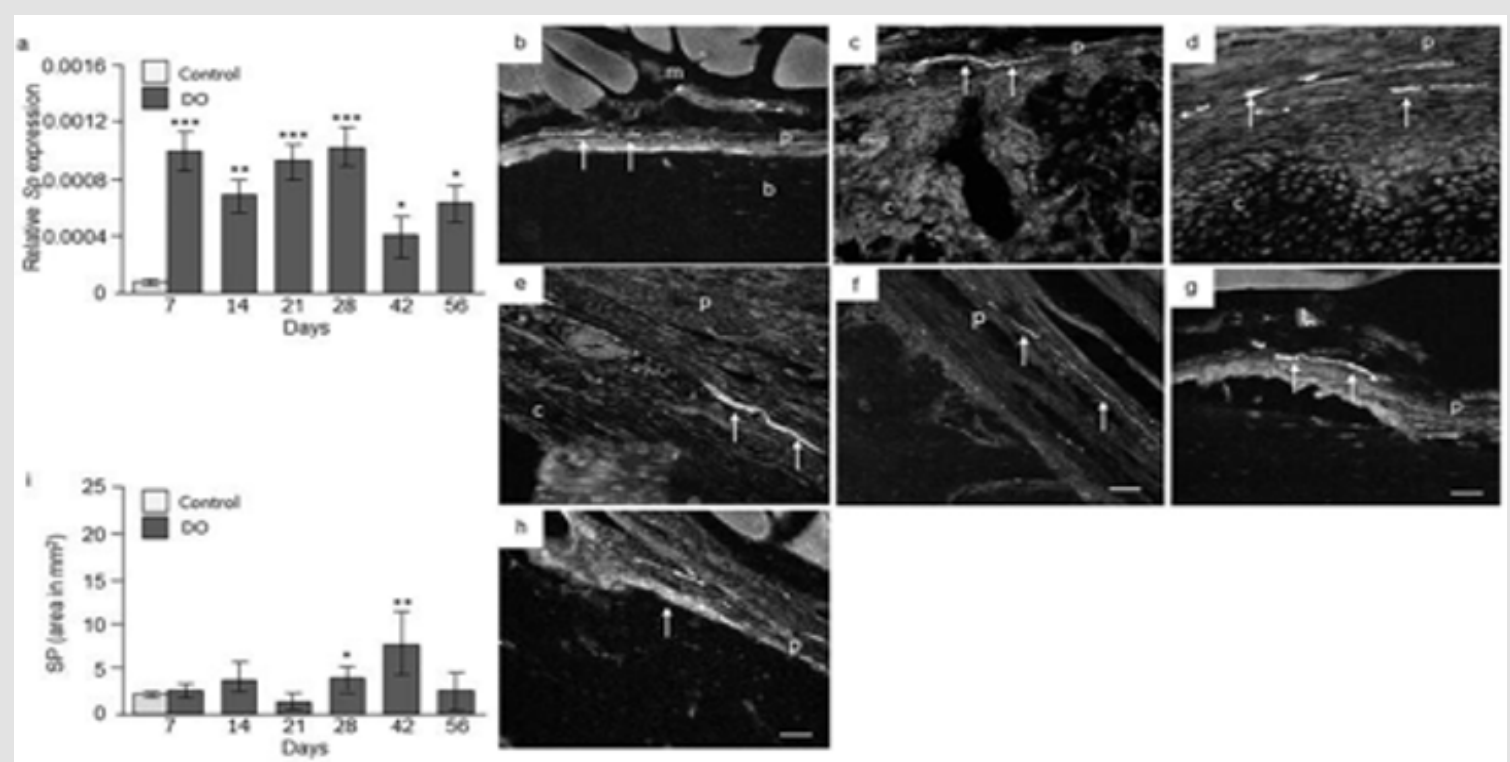

Figure 5: SP expression in control and distracted femur. a) Relative Sp gene expression at day 7, 14, 21, 28, 42, and 56 from distraction osteogenic (DO) and intact control (only day 7) femur. Values are expressed as mean \pm SEM of 5 rats per group. ${ }^{*} \mathrm{P}$ $\leq 0.05,{ }^{* *} \mathrm{p} \leq 0.005$ and ${ }^{* * *} \mathrm{P} \leq 0.0005$, compared to intact controls by ANOVA followed by Fisher's protected least significant difference test. Photomicrographs of b) intact control, and c) distracted femur at day 7; d) at day 14; e) day 21; f) day 28; g) day 48 , and h) day 56 stained with antibodies against SP. Original magnification is $20 \mathrm{x}$ and bars $=100 \mu \mathrm{m}$. $(\mathrm{m}=\mathrm{muscle}, \mathrm{p}=$ periosteum, $\mathrm{b}=$ bone, $\mathrm{c}=$ callus, $\mathrm{bv}=$ blood vessel). i) Semi-quantitative analysis of SP immunoreactivity (immunofluorescent area in $\mathrm{mm} 2$ ) at different time points from intact control and distraction osteogenic (DO) femur. Values are expressed as mean \pm SEM of 4 rats per group. ${ }^{*} \mathrm{P} \leq 0.05$ and ${ }^{* *} \mathrm{p} \leq 0.005$, compared to intact controls by ANOVA followed by Fisher's protected least significant difference test.

CGRP Expression: Quantitative RT-PCR analysis revealed a 9 fold [ $p=0.0004]$ increase in CGRP mRNA at day 7, a 6 fold increase $[p=0.0003]$ at day 14,3 fold increase $[p=0.0001]$ at day 21,4 fold increase [ $p=0.02]$ at day 28 and 3 fold increase [ $=0.04]$ at day 42 was observed in distracted portion of femur compared to intact control femur. No significant changes in CGRP mRNA were observed at day 56 in distracted portion of femur compared with control femur (Figure 6a). IHC analysis of intact control femur demonstrated no immunoreactivity within the bone. Thick nerve fibers positive to CGRP in close proximity to bone were observed at the periosteum at day 7 (Figure 6b). Abundant CGRP positive nerve fibers were seen in the periosteum, bone marrow, surrounding muscles adjacent to osteotomy at days 7 and 14, while few were observed at day 21. CGRP positive cells were observed in the periosteum, in and around the callus at day 7 and 14 (Figure 6c-6e). At day 28 and 42, an up rise in CGRP positive fibers were observed in the deep layer of hypertrophic periosteum with few fibers located inside the callus (Figure $6 \mathrm{f} \& 6 \mathrm{~g}$ ). At day 48 and 56, abundant CGRP positive fibers were observed in the periosteum especially around blood vessels, cortical bone and bone marrow adjacent to osteotomy. Some nerve fibers were arranged along blood vessels and others were distributed as free nerve terminals, especially nerve terminals were identified in cartilaginous callus and new woven bone in distraction portion (Figure 6h). Computerized image analysis of CGRP positive immunoreactivity in distracted and intact demonstrated significant increase in CGRP expression at day 14 [p = 0.04] while decreased CGRP immunoreactivity was observed at day 21 [ $p=0.04]$ with 5.6 $\mathrm{mm}$ distraction compared to intact control rat femur (Figure 6i). 


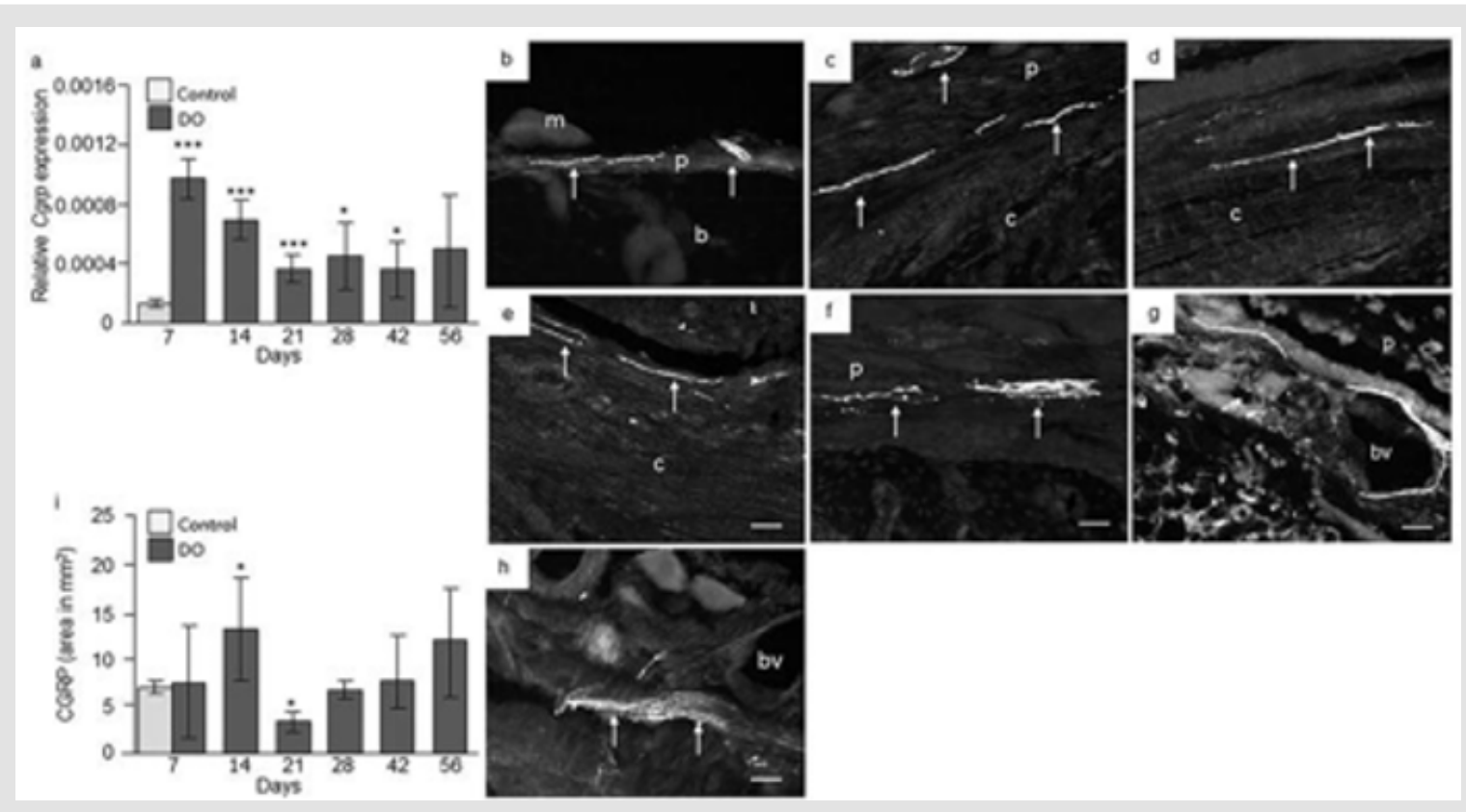

Figure 6: CGRP expression in control and distracted femur. a) Relative Cgrp gene expression at day 7, 14, 21, 28, 42, and 56 from distraction osteogenic (DO) and intact control (only day 7) femur. Values are expressed as mean \pm SEM of 5 rats per group. ${ }^{*} \mathrm{P} \leq$ 0.05 , and ${ }^{* * *} \mathrm{P} \leq 0.0005$, compared to intact controls by ANOVA followed by Fisher's protected least significant difference test. Photomicrographs of b) intact control, and c) distracted femur at day 7; d) at day 14; e) day 21; f) day 28; g) day 48, and h) day 56 stained with antibodies against CGRP. Original magnification is $20 x$ and bars $=100 \mu \mathrm{m} .(\mathrm{m}=$ muscle, $p=$ periosteum, $b=$ bone, $\mathrm{c}=$ callus, bv = blood vessel). i) Semi-quantitative analysis of CGRP immunoreactivity (immunofluorescent area in mm2) at different time points from intact control and distraction osteogenic (DO) femur. Values are expressed as mean \pm SEM of 4 rats per group. ${ }^{*} \mathrm{P} \leq 0.05$ compared to intact controls by ANOVA followed by Fisher's protected least significant difference test.

\section{Discussion}

Main findings of present studies are that during the initial period of bone fracture healing intense nerve regeneration occurs in the distraction area. With the maturation of bone tissues in the distracted part, nerve regeneration decreased with another episode of up regulation of nerve fibers during the bone remodeling stage. Mature nerve fibers were observed at later stages of bone healing when the bone architecture was attaining the normal structure in distracted gap. Thus, these results support the pertinent role of peripheral nervous system in bone fracture healing. The study also identified the up-regulated $S p$ and Cgrp mRNA levels within the distracted area, and determined increased CGRP positive nerve fibers during the early inflammatory, while SP positive nerve fibers predominantly, at the late modulating phase of bone healing. Generally, there are three main stages of fracture healing; inflammation, proliferation or repair and remodeling [21]. We observed intense nerve regeneration during early inflammatory phase of bone repair mainly in the hypertrophic periosteum and callus indicating crucial role of nervous system in early healing processes.

Nervous system is reported to play active role in healing by enhancing the proliferation of macrophages, fibroblasts, masts and endothelial cells which in turn secrete numerous cytokines and growth factors [22-25]. Macrophages and fibroblasts invade the injured site mainly during the early phases of healing [22,23]. Our observations of nerve regeneration during inflammatory phase highlight the probable neuronal influence on recruitment of macrophages or fibroblasts to the injury site, and to release the inflammatory mediators essential in healing during DO. Furthermore, the mechanical forces applied in DO can initiate the cascade of biologic processes of cellular differentiation, angiogenesis, and bone remodeling involving numerous inflammatory cytokines as well as growth factors $[4,5]$ most probably released by macrophages and fibroblasts. The release of these factors during DO can also produce a continuous stimulus on nerve regeneration as observed in present study. However, further studies are needed to delineate the exact role of nerve regeneration on inflammatory processes underlying fracture healing.

We observed mature nerve fibers especially in late bone modulating phase. These observations were strengthened by radiographic analysis and callus index. Callus index is a useful measure for quantifying bone formation and was maximum on day 56 indicating an active healing process. Moreover, higher levels of Runx2 gene expression were observed between day 14 to 56, when bone architecture most likely was attaining the normal structure in the distracted gap. Runx2 is a transcriptional factor involved in bone formation during the skeletal development [26]. Mature nerves probably stimulate alignments of bone cells, mineralization of bone matrix and regulate bone metabolism. The presence of nerves in the periosteum, bone marrow cavity and in vascular canals in mature long bone as well as in developing animals [27] 
probably endorse this assumption. A significant increase in $\mathrm{Sp}$ and Cgrp gene expression was observed during all stages of DO indicating synthesis of these peptides by local cells. Further, we observed SP- and CGRP- positive nerve fibers in and around callus, especially in close proximity to the distraction area indicating their role in osteogenesis.

The release of SP or CGRP from sensory nerves or from resident bone cells into the callus can potentially upregulate the production of matrixdegrading enzymes and sensitize ingrowing nerves and also enhance osteogenesis by effecting the collagen remodeling. Indeed, it has been shown that SP can increase collagen remodeling by enhancing the matrix metalloproteinase (MMP) -3 expression [28]. SP and CGRP and their receptors are reported on osteoblasts, osteoclasts and stem cells [29,30] and probably can induce osteogenesis by autocrine and/or paracrine fashion. Both SP and CGRP are reported to induce osteoblastic differentiation by acting through their receptors of bone marrow stromal stem cells (BMSCs) [28]. Production of SP and CGRP by mast cells, endothelial and stem cells have been reported [31]. Further, SP has been shown to stimulate the recruitment of stem cells to the site of injury through its receptor neurokinin 1 (NK1) [32]. SP along with nerve growth factor (NGF) is reported to promote tissue repair especially by enhancing angiogenesis [33]. Interestingly, NGF stimulates the expression of SP in nociceptive sensory neurons [34].

In consistence, we observed SP- and CGRP- positive nerve fibers around blood vessels in hypertrophic periosteum. Taken together, our findings of cellular and neuronal SP and CGRP expression in the distracted area specify their role in bone formation as well in angiogenesis. We further observed abundant SP positive nerve fibers in non-vascular cartilaginous callus and new woven bone as free nerve terminals indicating crucial role of SP in bone formation. SP has been shown to stimulate recruitment of stem cells to the site of injury [30]. Further, it has been reported that SP can stimulate bone colony formation, in-vitro, most probably by stimulating osteoblastic activity or regulating the osteoprogenitor cell differentiation [35]. Moreover, osteoblasts and osteoclasts which regulate bone formation and bone resorption express functional receptors for SP [15,35]. SP can promote connective tissue repair especially by enhancing angiogenesis [33]. Taken together, present and previous findings support the role of SP as a promoter or stimulator for new bone formation. The increased CGRP expression was noted in early inflammatory while SP in the late modulatory phase of healing.

CGRP released by central and peripheral nervous system influence the growth, repair and normal bone metabolism [36]. CGRP receptors are present on osteoblasts and octeoclasts and CGRP promote osteoblast differentiation and inhibit osteoclast formation directly by interacting with functional CGRP receptors expressed by these cells $[37,7]$. Further, it has been reported that magnesium induced neuronal CGRP contribute to bone formation after fracture healing in rats [38]. CGRP activate healing processes mainly by enhancing angiogenesis, epidermal regeneration, and recruitment of endothelial precursors at the fracture site $[39,40]$. Moreover, present findings confirm our previous observations of more CGRP-containing nerve fiber in the osteogenic regions of bone such as periosteum and epiphysis and less in the cortical area [41]. Furthermore, the specific denervation of sensory nerves system by capsaicin treatment or surgical denervation caused significant loss of trabecular bone integrity, reduced bone mass and strength in rat bone. These changes were closely related to the local content of SP and CGRP $[42,43]$.

The animal model employed in this study is a well characterized and reproducible model of bone distraction and is frequently been used in bone repair and fracture healing research $[17,18]$. DO followed by four weeks of latency phase presented all stages of bone formation in one and same bone for the comparative analysis of GAP-43, PGP 9.1, SP and CGRP expression at different time points during healing. Taken together, our present findings provide an insight that nerve regeneration with sensory nerve into the distracted gap of fractured bone, probably are crucial for early tissue healing and presumably a prerequisite for osteogenesis and later bone modeling. Local delivery of nerve regenerative mediators or sensory neuropeptides might be a good approach to accelerate bone consolidation to reduce the time required for fracture healing.

\section{Funding Sources and Role}

The research leading to these results has received funding from the Swedish Medical Research Council [13107, MA]; and the A0/ASIF Foundation, Switzerland [05-A67, MA]. There was no role for any funding agency regarding the study design, collection, and analysis, interpretation of data, manuscript writing or in the decision to submit the manuscript for publication.

\section{Competing Interests}

There are no professional or financial conflicts of interests to disclose.

\section{References}

1. Aldegheri R (1999) Distraction osteogenesis for lengthening of the tibia in patients who have limblength discrepancy or short stature. The Journal of bone and joint surgery American volume 81(5): 624-34.

2. Aronson J, Shin HD (2003) Journal of pediatric orthopedics Imaging techniques for bone regenerate analysis during distraction osteogenesis.23(4): 550-560.

3. Li G, Simpson AH, Kenwright J, Triffitt JT (1997) Assessment of cell proliferation in regenerating bone during distraction osteogenesis at different distraction rates. Journal of orthopaedic research: official publication of the Orthopaedic Research Society 15(5): 765-772.

4. Cope JB, Samchukov ML (2000) Regenerate bone formation and remodeling during mandibular osteodistraction. The Angle orthodontist 70(2): 99-111.

5. Zhao D, Wang Y, Han D ( 2016) Periosteal Distraction Osteogenesis: An Effective Method for Bone Regeneration. Biomed Res Int 2075317 doi $10.1155 / 2016 / 2075317$. 
6. Li J, Ahmad T, Spetea M, Ahmed M, Kreicbergs A (2001) Bone reinnervation after fracture: a study in the rat. Journal of bone and mineral research : the official journal of the American Society for Bone and Mineral Research 16(8): 1505-1510.

7. Wang L, Shi X, Zhao R, Halloran BP, Clark DJ, et al. ( 2010) Calcitoningene-related peptide stimulates stromal cell osteogenic differentiation and inhibits RANKL induced NF-kappaB activation, osteoclastogenesis and bone resorption. Bone 46(5): 1369-1379.

8. Offley SC, Guo TZ, Wei T, Clark JD, Vogel H, et al. (2005) Capsaicinsensitive sensory neurons contribute to the maintenance of trabecular bone integrity. Journal of bone and mineral research: the official journal of the American Society for Bone and Mineral Research 20(2): 257-267.

9. Cherruau M, Morvan FO, Schirar A, Saffar JL (2003) Chemical sympathectomy-induced changesin TH-, VIP-, and CGRP-immunoreactive fibers in the rat mandible periosteum: influence on bone resorption Journal of cellular physiology 194(3): 341-348.

10. Konttinen Y, Imai S, Suda A (1996) Neuropeptides and the puzzle of bone remodeling. State of the art. Acta orthopaedica Scandinavica 67(6): 632639.

11. Bjurholm A, Kreicbergs A, Schultzberg M, Lerner UH (1992) Neuroendocrine regulation of cyclic AMP formation in osteoblastic cell lines (UMR-106-01, ROS 17/2.8, MC3T3-E1, and Saos-2) and primary bone cells. Journal of bone and mineral research: the official journal of the American Society for Bone and Mineral Research 7(9): 1011-1019.

12. Vignery A, McCarthy TL (1996) The neuropeptide calcitonin generelated peptide stimulates insulinlike growth factor I production by primary fetal rat osteoblasts Bone 18(4): 331-335

13. Cornish J, Callon KE, Lin CQ Xiao CL, Gamble GD, Cooper GJ, et al. (1999) Comparison of the effects of calcitonin gene-related peptide and amylin on osteoblasts. Journal of bone and mineral research: the official journal of the American Society for Bone and Mineral Research 14(8): 13021309.

14. Wang L, Zhao R, Shi X, Wei T, Halloran BP, et al. (2009) Substance P stimulates bone marrow stromal cell osteogenic activity, osteoclast differentiation, and resorption activity in vitro Bone 45(2): 309-320.

15. Elefteriou F (2008) Regulation of bone remodeling by the central and peripheral nervous system. Archives of biochemistry and biophysics 473(2): 231-236

16. Zhang YB, Wang L, Jia S, Du ZJ, Zhao YH, et al. (2014) Local injection of substance $\mathrm{P}$ increases bony formation during mandibular distraction osteogenesis in rats. The British journal of oral \& maxillofacial surgery 52(8): 697-702.

17. Mark H, Bergholm J, Nilsson A, Rydevik B, Stromberg L (2003) An external fixation method and device to study fracture healing in rats. Acta orthopaedica Scandinavica 74(4): 476-482.

18. Mark H, Nilsson A, Nannmar u, Rydevik B (2004) Effects of fracture fixation stability on Ossification in healing fractures Clin Orthop I (419): 244-250.

19. Isefuku S, Joyner CJ, Reed AA, Simpson AH (2004) Distraction osteogenesis in the Cbfa-1+/- mouse. Journal of orthopaedic research: official publication of the Orthopaedic Research Society 22(6): 12761282

20. Welch RD, Birch JG, Makarov MR, Samchukov ML (1998) Histomorphometry of distraction osteogenesis in a caprine tibial lengthening model. Journal of bone and mineral research: the official journal of the American Society for Bone and Mineral Research 13(1): $1-9$

21. Nyari T, Scamell BE (2015) Principles of bone and joint injuries and their healing. Surgery(Oxford) 33(1): 7-14.

22. He L, Marneros AG (2013) Macrophages are essential for the early wound healing response and the formation of a fibrovascular scar. Am J Pathol 182(6): 2407-2417
23. Zhang H, Kot A, Lay YE, Fierro FA, Chen H Lane NE, Yao W (2017) Acceleration of Fracture Healing by Overexpression of Basic Fibroblast Growth Factor in the Mesenchymal Stromal Cells. Stem Cells Transl Med 6(10): 1880-1893.

24. Yamamoto K, Yamamoto T, Honjo K, Ichioka H, Oseko F, et al. (2015) Electrical stimulation with periodic alternating intervals stimulates neuronal cells to produce neurotrophins and cytokines through activation of mitogen-activated protein kinase pathways. Eur J Oral Sci 123(6): 403-408

25. Chéret J, Lebonvallet N, Buhé V, Carre JL, Misery L, et al. (2014) Influence of sensory neuropeptides on human cutaneous wound healing process. J Dermatol Sci 74(3): 193-203.

26. McGee-Lawrence ME, Carpio LR, Bradley EW, Dudakovic A, Lian JB, et al. (2014) Runx2 is required for early stages of endochondral bone formation but delays final stages of bone repair in Axin2-deficient mice. Bone 66: 277-286.

27. Sisask G, Bjurholm A, Ahmed M, Kreicbergs A (1995) Ontogeny of sensory nerves in the developing skeleton. The Anatomical record 243(2): 234-240.

28. Fong G,Backman L.J,Hart D. A,Danielson P, McCormack B, Scott A (2013) Substance $\mathrm{P}$ enhances collagen remodeling and MMP-3 expression by human tenocytes. Journal of orthopaedic research: official publication of the Orthopaedic Research Society 31(1): 91-98.

29.Zhou R, Yuan Z, Liu J, Liu J (2016) Calcitonin gene-related peptide promotes the expression of osteoblastic genes and activates the WNT signal transduction pathway in bone marrow stromal stem cells. Mol Med Rep 13(6): 4689-4696.

30. Wang L, Zhao R, Shi X, Wei T, Halloran BP, et al. (2009) Substance P stimulates bone marrow stromal cell osteogenic activity, osteoclast differentiation, and resorption activity in vitro. Bone 45(2): 309-320.

31. Bulut K, Felderbauer P, Deters S, Hoeck K, Schmidt Choudhury A, et al. (2008) Sensory neuropeptides and epithelial cell restitution: the relevance of SP- and CGRP-stimulated mast cells. Int J Colorectal Dis 23(5): 535-541.

32. Hong HS, Lee J, Lee E, Kwon YS, Lee E, et al. (2009) A new role of substance $\mathrm{P}$ as an injuryinducible messenger for mobilization of CD29(+) stromallike cells. Nature medicine 15(4): 425-435.

33. Seegers HC, Hood VC, Kidd BL, Cruwys SC, Walsh DA, et al. (2003) Enhancement of angiogenesis by endogenous substance $P$ release and neurokinin-1 receptors during neurogenic inflammation. The Journal of pharmacology and experimental therapeutics 306(1): 8-12.

34. Kepler CK, Markova DZ, Hilibrand AS, Vaccaro AR, Risbud MV, et al. (2013) Substance P stimulates production of inflammatory cytokines in human disc cells. Spine (Phila Pa 1976) 38(21): 1291-1299.

35. Shih C, Bernard GW (1997) Neurogenic substance P stimulates osteogenesis in vitro. Peptides 18(2): 323-326.

36. Xu G, Jiang D (2014) The role and mechanism of exogenous calcitonin gene-related peptide on mesenchymal stem cell proliferation and osteogenetic formation. Cell Biochem Biophys 69(2): 369-378.

37. Nakamura M, Morimoto S, Yang Q Hisamatsu T, Hanai N, et al. (2005) Osteoclast-like cells express receptor activity modifying protein 2 : application of laser capture microdissection. J Mol Endocrinol 34(1): 257-261.

38. Zhang Y, Xu J, Ruan YC, Yu MK, O Laughlin M, et al. (2016) Implantderived magnesium induces local neuronal production of CGRP to improve bone-fracture healing in rats. Nat Med 22(10): 1160-1169.

39. Brain SD, Grant AD (2004) Vascular actions of calcitonin gene-related peptide and adrenomedullin. Physiological reviews 84(3): 903-934.

40. Tuo Y, Guo X, Zhang X, Wang Z, Zhou J, et al. (2013) The biological effects and mechanisms of calcitonin gene-related peptide on human endothelial cell. J Recept Signal Transduct 33: 114-123. 
41. Li J, Kreicbergs A, Bergström J, Stark A, Ahmed M, et al. (2007) Sitespecific CGRP innervation coincides with bone formation during fracture healing and modeling: A study in rat angulated tibia. J Orthop Res 25(9): 1204-1212.

42. Ahmed M, Bjurholm A, Srinivasan GR, Lundeberg T, Theodorsson E, et al. (1995) Capsaicin effects on substance P and CGRP in rat adjuvant arthritis. Regul Pept 55(1): 85-102.

ISSN: 2574-1241

DOI: 10.26717/BJSTR.2019.22.003797

Aisha Ahmed. Biomed J Sci \& Tech Res

(c) (P) This work is licensed under Creative

Submission Link: https://biomedres.us/submit-manuscript.php
43. Ahmed M, Srinivasan GR, Theodorsson E, Schultzberg M, Kreicbergs A, et al. (1995) Effects of surgical denervation on substance $P$ and calcitonin gene-related peptide in adjuvant arthritis. Peptides 16(4): 56979.

$\begin{array}{ll}\text { BIOMEDICAL } & \text { Assets of Publishing with us } \\ \text { RESEARCHES } & \text { - Global archiving of articles } \\ & \text { - Immediate, unrestricted online access } \\ & \text { - Rigorous Peer Review Process } \\ & \text { - Authors Retain Copyrights } \\ \end{array}$

